\title{
Bickerstaff's brainstem encephalitis associated with typhoid fever
}

\author{
G.M. Wali
}

Department of Neurology, K.L.E. Society's Hospital and Medical Research Centre, Nehru Nagar, Belgaum - 590 010, Karnataka State, India

\begin{abstract}
Summary: A 14 year old boy developed the syndrome of Bickerstaff's brainstem encephalitis during the course of bacteriologically proved typhoid fever. The clinical course and the results of various neurological investigations are detailed. This report adds a further manifestation to the published neuropsychiatric complications of typhoid fever.
\end{abstract}

\section{Introduction}

The various neuropsychiatric manifestations of typhoid fever include delirium, semi-coma, coma, meningitis, convulsions, generalized myoclonus, focal neurological deficits, transient parkinsonism, motor neurone disorder, symmetrical sensory motor polyneuropathy, mononeuritis, schizophrenic psychoses ${ }^{1}$ and cerebellar syndrome. ${ }^{2}$ In this communication a boy who developed the syndrome of brainstem encephalitis during the course of typhoid fever is described. To the best of my knowledge this is the first case report of this association.

\section{Case report}

A 14 year old boy was seen in January 1989 with a 8-day history of continuous fever with chills and headache. Four days before admission he developed diplopia, difficulty in swallowing and nasal twang to his speech. Over the next 2 days his condition deteriorated with the appearance of nasal regurgitation and gait ataxia. On admission, he was found to be febrile $\left(101^{\circ} \mathrm{F}\right)$, mildly dehydrated and to have mild hepatosplenomegaly. $\mathrm{He}$ was drowsy but responding relevantly to verbal commands. He had bilateral ptosis and loss of upward and horizontal conjugate eye movements. Downward movement was partially restricted. The pupils and fundi were normal. There was bilateral palatal weakness and speech was dysarthric with a nasal twang. Facial and masticatory muscles were not involved. He had a moderate degree of inco-

Correspondence: G.M. Wali, M.D., D.M. Accepted: 22 April 1991 ordination of all the limbs with gross gait ataxia. Motor power was normal and there was no sensory deficit. Generalized areflexia was present. Plantar reflexes were not elicitable. There was mild neck stiffness. Over the first 4 days in hospital bulbar weakness increased leading to an almost aphonic state, truncal ataxia appeared and he needed help to sit erect.

Haematological studies and blood chemistry were normal except for an ESR of $30 \mathrm{~mm} / \mathrm{h}$. Blood Widal done on the day of admission was positive, showing 1:160 titre for the $O$ antigen and 1:320 titre for the $\mathbf{H}$ antigen. Repeat Widal a week later showed the titres for both antigens at 1:320. Blood culture grew Salmonella typhi. At lumbar puncture, cerebrospinal fluid (CSF) study showed no cells, normal sugar and normal protein. It was negative for culture of organisms, acid fast bacilli, fungi and VDRL. A computed tomographic scan of the head was normal. An encephalogram (EEG) done on the 2nd day showed bilateral diffuse delta slowing. Bilateral auditory evoked potentials were normal. Nerve conduction velocities, including the F-wave responses, were normal.

He was treated with a combination of intravenous ampicillin and oral trimethoprim-sulphamethoxazole for 10 days. The temperature returned to normal on the 4th day of admission and a day later he became fully conscious. Ptosis disappeared and eye movements became normal within a week. The gait, swallowing and speech improved over the next 2 weeks although he was left with a mild nasal twang. A repeat EEG done 2 weeks after the first one showed bilateral diffuse theta slowing while the 3rd one, done one month after clinical recovery, about 2 months after the first EEG, was normal. When reviewed one year later he was well except for the nasal twang. 


\section{Discussion}

The syndrome of brainstem encephalitis was described by Bickerstaff and Cloake in $1951 .{ }^{3}$ It consists of subacute onset brainstem dysfunction which generally appears after a few days of malaise and headache which are presumed to be of viral aetiology. The patient may appear moribund at the height of the neurological syndrome but generally recovers well. This has prompted Bickerstaff ${ }^{4}$ to call it a grave syndrome with a benign prognosis. A study of reported cases indicates the possibility of more than one mechanism being involved in the production of the syndrome. Amir Najim Al-Din and others, ${ }^{5}$ who reported a series of 18 cases, suspect it to be a 'hypersensitivity' or 'allergic' response of the central nervous system to some external and probably infective challenge. A similar explanation is given by Dhir et al. ${ }^{6}$ who reported a case of brainstem encephalitis following acute haemorrhagic conjunctivitis. Direct invasion of the central nervous system has been shown to be the cause in case of herpetic brainstem encephalitis ${ }^{7}$ and Listeria rhomboencephalitis. ${ }^{8}$ Typhoid fever was confirmed in the present case by blood culture and serology. A normal computed tomographic scan of the head and normal CSF have ruled out the possibility of direct infection of the central nervous system or CSF. I consider the possibility of a toxic effect of $S$. typhi endotoxin on the brainstem as the possible mechanism in the present case. A similar explanation has been given by Osuntokun et $a .^{1}{ }^{1}$ to account for the several neurological complications of typhoid fever.

\section{Acknowledgement}

I thank Mr S.B. Nesargi for technical assistance.

\section{References}

1. Osuntokun, B.O., Bademosi, O., Ogunremi, K. \& Wright, S.G. Neuropsychiatric manifestations of typhoid fever in 959 patients. Arch Neurol 1972, 27: 7-13.

2. Wadia, R.S., Ichaporia, N.R., Kiwalkar, R.S., Amin, R.R. \& Sardesai, H.V. Cerebellar ataxia in enteric fever. $J$ Neurol Neurosurg Psychiatry 1985, 48: 695-697.

3. Bickerstaff, E.R. \& Cloake, P.C.P. Mesencephalitis and rhombencephalitis. Br Med J 1951, 2: 77-81.

4. Bickerstaff, E.R. Brainstem encephalitis - further observations on a grave syndrome with benign prognosis. $\mathrm{Br}$ Med J 1957, 1: 1384-1387.

5. Amir, N.A., Anderson, M., Bickerstaff, E.R. \& Harvey, I Brainstem encephalitis and the syndrome of Miller Fischer, a clinical study. Brain 1982, 105: 481-495.

6. Dhir, S.P., Munjal, V.P. \& Jain, K.S. Bickerstaff's encephalitio following acute haemorrhagic conjunctivitis. Indian JOphthalmol 1984, 32: 45-47.

7. Roman-Campos, G. \& Toro, G. Herpetic brainstem encephalitis. Neurology 1980, 30: 981-985.

8. Weinstein, A.J., Schiavone, W.A. \& Furlan, A.J. Listeria rhombencephalitis. Arch Neurol 1982, 39: 514-516. 